\title{
Patient Perspectives on Combination Therapy of a Once-weekly Oral Medication Plus Daily Medication for Lifestyle-related Chronic Diseases
}

\author{
Mitsuyoshi Takahara ${ }^{1,2}$, Toshihiko Shiraiwa ${ }^{3}$, Naoko Ogawa ${ }^{4}$, Mayumi Yamamoto ${ }^{4}$, \\ Yuko Kusuda ${ }^{3}$, Megumi Shindo ${ }^{3}$, Saki Hashio ${ }^{3}$, Naoto Katakami ${ }^{2}$, \\ Taka-aki Matsuoka ${ }^{2}$ and Iichiro Shimomura ${ }^{2}$
}

\begin{abstract}
Objective The current study investigated whether or not patients taking multiple daily oral medications for lifestyle-related chronic diseases would have positive perspectives on changing one of their medications to a once-weekly one.

Methods A total of 1,071 Japanese outpatients participated in the current study. We performed a questionnaire-based survey and compared the current satisfaction with the ongoing daily oral treatment (current daily-only treatment) and an expected satisfaction with an imaginary oral treatment changing one of their daily oral medications to a once-weekly oral medication (imaginary daily-and-weekly treatment).

Results Medications were taken for diabetes mellitus in $72 \%$ of the patients, for dyslipidemia in $54 \%$, and for circulatory diseases, including hypertension, in $73 \%$. Compared to their satisfaction with the current dailyonly treatment, an expected satisfaction with the imaginary daily-and-weekly treatment was on average significantly attenuated $(\mathrm{p}<0.001$, effect size $\mathrm{d}=0.49)$. The prevalence of a higher satisfaction score for the imaginary daily-and-weekly treatment versus the current daily-only treatment was $30 \%$ in the overall population. The prevalence was $59 \%, 40 \%, 29 \%, 14 \%$, and $8 \%$ in the 1st, 2nd, 3rd, 4 th, and 5th quintile of the satisfaction score with the current daily-only treatment ( $\mathrm{p}<0.001$ for trend).

Conclusion Treatment satisfaction would be on average attenuated if one of the multiple daily oral medications was changed to a once-weekly one. Improvement in the satisfaction was less expected in the subgroup that was more satisfied with the current daily-only treatment.
\end{abstract}

Key words: once-weekly medication, patient perspective, lifestyle-related chronic disease

(Intern Med 56: 615-620, 2017)

(DOI: 10.2169/internalmedicine.56.7583)

\section{Introduction}

Patients with lifestyle-related chronic diseases, including diabetes mellitus, dyslipidemia, and hypertension, are often treated with daily oral medications, and adherence to the medications is a key factor in the management of chronic diseases $(1,2)$. It is well recognized that patients' satisfaction with treatment is a major determinant of adherence (3), and strategies for improving treatment satisfaction have been long discussed. One often suggested strategy is to lessen dosing frequency. Previous studies have reported favorable findings for decreased daily dosing frequency (4). Furthermore, on the basis of the belief that a lower dosing frequency would improve satisfaction, once-weekly oral medications have been developed for lifestyle-related chronic diseases. Recently, a few of them have begun to make an appearance in clinical practice (5-7).

${ }^{1}$ Department of Metabolic Medicine, Osaka University Graduate School of Medicine, Japan, ${ }^{2}$ Department of Diabetes Care Medicine, Osaka University Graduate School of Medicine, Japan, ${ }^{3}$ Shiraiwa Medical Clinic, Shiraiwa Medical Clinic, Japan and ${ }^{4}$ Smile Pharmacy, Japan Received for publication April 13, 2016; Accepted for publication June 23, 2016 Correspondence to Dr. Mitsuyoshi Takahara, takahara@endmet.med.osaka-u.ac.jp 
It is true that once-weekly bisphosphonates, an antiosteoporotic drug, was a successful precedent for onceweekly oral medications. As previous studies revealed, osteoporotic patients strongly preferred a once-weekly bisphosphonate to a once-daily one $(8,9)$. However, bisphosphonates are far from easy to take, whereas medications for lifestyle-related chronic diseases currently available are not as troublesome as bisphosphonates. It might be that the troublesomeness of taking bisphosphonates encourages the preference for once-weekly dosing versus more frequent dosing. It remains unknown whether this preference would be similarly true for less troublesome medications for lifestyle-related chronic diseases.

In addition, given that only a few kinds of once-weekly medications are available at present, and that patients with lifestyle-related chronic diseases often take a number of oral medications, it is currently impossible to change all of their medications to once-weekly ones. As such, any once-weekly medications must be taken together with daily medications. However, little is known about the patient perspectives on the combined use of a once-weekly medication with daily medications.

In the present study, we examined whether changing a daily oral medication for a once-weekly oral one would improve treatment satisfaction in patients treated with more than one daily oral medications for chronic diseases. We performed a questionnaire-based survey, to compare the current satisfaction with the ongoing daily oral treatment (current daily-only treatment) and an expected satisfaction with an imaginary oral treatment changing one of the daily oral medications for a once-weekly oral medication (imaginary daily-and-weekly treatment).

\section{Materials and Methods}

\section{Questionnaire}

The current questionnaire was developed to measure satisfaction with oral treatment. The questionnaire contained a total of 10 statements related to treatment satisfaction and asked to what extent a respondent agreed with each statement. The response was obtained on a 7-point Likert scale, with a range from 0 , indicating "strongly disagree", to 6 , indicating "strongly agree". The 10 statements consisted of 6 positively and 4 negatively worded ones. The positive statements concerned convenience (item no. 1), encouragement of adherence (no. 2), control over diseases (no. 3), feeling of healthiness (no. 4), satisfaction (no. 9), and hope for receiving the treatment (no. 10). The negative statements concerned troublesomeness (no. 5), fear of forgetting to take medicines (no. 6), suspicion about efficacy (no. 7), and unfavorably weakened disease awareness (no. 8). The questionnaire was developed on the concept that all items would, either positively or negatively, reflect a single underlying factor, namely satisfaction with oral treatment, and calculation of a total score was expected.
Regarding the current daily-only treatment, the questionnaire asked how a respondent felt about his or her current daily oral treatment, with the following 10 statements presented:

1. The current oral treatment is convenient.

2. The current oral treatment encourages adherence.

3. The current oral treatment controls my diseases well.

4. The current oral treatment makes me feel healthy.

5. The current oral treatment is troublesome.

6. The current oral treatment makes me afraid of forgetting to take medicines.

7. I am suspicious about the efficacy of the current oral treatment.

8. The current oral treatment undesirably weakens my disease awareness.

9. I am satisfied with the current oral treatment.

10. I hope to continue receiving the current oral treatment.

Similarly, to examine the expected satisfaction with an imaginary daily-and-weekly treatment, the questionnaire asked, "If you could change only one of your daily oral medications for a once-a-week oral medication, how would you feel about the oral treatment including the weekly medication?," with the following 10 statements presented:

1. The oral treatment will be convenient.

2. The oral treatment will encourage adherence.

3. The oral treatment will control my diseases well.

4. The oral treatment will make me feel healthy.

5. The oral treatment will be troublesome.

6. The oral treatment will make me afraid of forgetting to take medicines.

7. I will be suspicious about the efficacy of the oral treatment.

8. The oral treatment will undesirably weaken my disease awareness.

9. I will be satisfied with the oral treatment.

10. I hope to try receiving the oral treatment.

Note that all of the statements for the current daily-only treatment and those for the imaginary daily-and-weekly treatment corresponded to each other.

\section{Study population and procedures}

The study population were adult Japanese outpatients with lifestyle-related chronic disease who had multiple daily oral medications prescribed in Shiraiwa Medical Clinic, Osaka, Japan. A total of 1,091 outpatients were asked to participate in the current study, and 1,071 (98\%) ultimately participated. The study was performed in accordance with the Declaration of Helsinki and was approved by the ethics committees of Shiraiwa Medical Clinic and Osaka University Hospital. Informed consent was obtained from every participant in the current study. The participants responded to the questionnaire for both the current daily-only treatment and an imaginary daily-and-weekly treatment. The patient attributes, including age, sex, and dosing regimens they re- 
Table 1. Characteristics of Study Population.

\begin{tabular}{lc}
\hline $\mathrm{n}$ & 1,071 \\
\hline Age (years) & $66 \pm 12$ \\
\hline Male sex & $623(58 \%)$ \\
\hline Medication for diabetes mellitus & $774(72 \%)$ \\
\hline Medication for dyslipidemia & $575(54 \%)$ \\
\hline Medication for circulatory diseases & $778(73 \%)$ \\
\hline Medication for hyperuricemia & $150(14 \%)$ \\
\hline Number (i.e., kind) of oral medications & $4.7 \pm 2.5$ \\
\hline Frequency of taking oral medications (times per day) & $3.0 \pm 1.7$ \\
\hline Combination with self-injections & $318(30 \%)$ \\
\hline
\end{tabular}

Data are mean \pm standard deviation or number (percentage).

ceived, were collected from their medical records.

\section{Statistical analysis}

Descriptive statistics are given as the mean \pm standard deviation for continuous variables and as percentages for categorical variables if not otherwise mentioned. A $p$ value less than 0.05 was considered to be significant, and $95 \%$ confidence intervals (CIs) were given when required.

An explorative factor analysis with a varimax rotation was performed on the 10 items of the questionnaire. The Kaiser criterion, i.e. Eigenvalue $>1$, was used to determine the optimal number of factors. In a factor analysis, the good scaling property of each item was judged by loadings of $>0.40$ on one factor and $<0.30$ on any other factor. Communalities were also reported. The reliability of each structure was assessed with Cronbach's $\alpha$ and item-total correlations. For internal consistency, Cronbach's $\alpha$ of 0.80 or higher and an item-total correlation of 0.20 or higher were regarded as acceptable. Cronbach's $\alpha$ with a specific item deleted was evaluated too.

The difference in the scores between the current dailyonly treatment and an imaginary daily-and-weekly treatment was tested by paired $t$ test. Effect size $d$ was reported together with the $\mathrm{p}$ value when needed. Correlation was assessed using Pearson's correlation coefficient $r$. A p value for trend was obtained by one-way analysis of variance for continuous variables and by the linear-by-linear association test for dichotomous variables. The general linear model was developed to explore the independent association of dosing regimens with treatment satisfaction after adjustment for patient attributes, including age, sex, and chronic diseases for which medications were prescribed. All statistical analyses were performed using the IBM SPSS Statistics Version 22 software program (SPSS Inc., Chicago, IL, USA).

\section{Results}

Patient characteristics are shown in Table 1. Complete responses to the questionnaire for the current daily-only treat- ment were obtained in 1,022 of the 1,071 patients (95\%).

An exploratory factor analysis revealed 2 factors with Eigenvalue $>1$ (4.7 and 1.7). In the 2-factor structure, explaining $55 \%$ of variance, all 10 items were successfully separated into 6 positively and 4 negatively worded items (see 2-factor solution in Table 2). Their communalities ranged from 0.357 to 0.654 . Cronbach's $\alpha$ was 0.881 for the 6-item positively worded scale and 0.816 for the 4-item negatively worded scale, whereas the corresponding itemtotal correlations ranged from 0.562 to 0.744 and 0.617 to 0.669 , respectively. In contrast, in the 1-factor structure, explaining $42 \%$ of variance, all 10 items again had $>0.40$ of the absolute loading values; the loadings were $>0.40$ for the 6 positive items and $<-0.40$ for the 4 negative items (see 1 factor solution in Table 2). Their communalities ranged from 0.223 to 0.598 . In the assessment of reliability with the 4 negative items scored inversely, the item-total correlations ranged from 0.490 to 0.665 , and Cronbach's $\alpha$ was as high as 0.862 , which was never exceeded if any item was deleted (Table 2). These findings indicated a high internal consistency, suggesting that all 6 positive and 4 negative items related to one underlying factor, and that a total score of satisfaction could be developed by summing the scores of all 10 items, with the rating of the 4 negatively worded items reversed. This total satisfaction score had a possible range between 0 and 60 . The score was highly correlated with the factor score $(r=0.975)$.

In the study population, the satisfaction score for the current daily-only treatment was $43 \pm 11$ (median, 42; interquartile range, 34 to 52 ; range 8 to 60 ). The general linear model adjusted for patient attributes revealed that dosing regimens independently associated with the satisfaction scores were the frequency of dosing (standardized partial regression coefficient $\beta=-0.182, p<0.001)$ and the combined use of self-injections ( $\beta=-0.077, p=0.027)$, but not the number (i.e. the kind) of medications ( $\beta=-0.040, p=0.402$ ).

Of the 1,022 patients who completely responded to the questionnaire for the current daily-only treatment, 973 (95\%) also completely responded to that for an imaginary daily-and-weekly treatment. As Table 3 shows, the individual item scores for the imaginary daily-and-weekly treatment were worse on the whole than those for the current daily-only treatment. The total satisfaction score, calculated similarly to the current daily-only treatment, was significantly lower than that for the current treatment, with $\mathrm{p}$ value less than 0.001 and effect size $d$ equal to 0.49 . Thirty percent of the patients $(n=291)$ gave a higher score for the imaginary daily-and-weekly treatment than for the current daily-only treatment.

As shown in Table 4, patients giving a higher score for the imaginary daily-and-weekly treatment than for the current daily-only treatment were more prevalent in the subgroup with a lower score for the current daily-only treatment. The general linear model revealed that none of the current dosing regimens were significantly associated with the satisfaction score for an imaginary daily-and-weekly 
Table 2. Factor Structure and Reliability of Questionnaire.

\begin{tabular}{|c|c|c|c|c|c|}
\hline \multirow{3}{*}{ Item } & \multicolumn{3}{|c|}{ Factor structure } & \multicolumn{2}{|c|}{ Reliability for 1-factor structure } \\
\hline & \multicolumn{2}{|c|}{ 2-factor solution } & \multirow{2}{*}{$\begin{array}{l}\text { 1-factor solution } \\
\text { Factor } 1\end{array}$} & \multirow{2}{*}{$\begin{array}{l}\text { Item-total } \\
\text { correlation }\end{array}$} & \multirow{2}{*}{$\begin{array}{l}\text { Chronbach's } \alpha \\
\text { if item deleted }\end{array}$} \\
\hline & Factor 1 & Factor 2 & & & \\
\hline 1. Convenience & 0.775 & -0.202 & 0.757 & 0.646 & 0.844 \\
\hline 2. Encouragement of adherence & 0.777 & -0.224 & 0.773 & 0.665 & 0.844 \\
\hline 3. Control over diseases & 0.697 & -0.225 & 0.711 & 0.621 & 0.848 \\
\hline 4. Feeling of healthiness & 0.577 & -0.155 & 0.572 & 0.490 & 0.856 \\
\hline 5. Troublesomeness & -0.250 & 0.669 & -0.555 & 0.579 & 0.850 \\
\hline 6. Fear of forgetting to take medicines & -0.152 & 0.672 & -0.472 & 0.496 & 0.858 \\
\hline 7. Suspicion about efficacy & -0.202 & 0.710 & -0.531 & 0.557 & 0.852 \\
\hline 8. Undesirably weakened disease awareness & -0.160 & 0.760 & -0.514 & 0.550 & 0.852 \\
\hline 9. Satisfaction & 0.769 & -0.195 & 0.748 & 0.646 & 0.845 \\
\hline 10. Hope for receiving the treatment & 0.750 & -0.165 & 0.714 & 0.602 & 0.847 \\
\hline
\end{tabular}

Two-factor solution explained $55 \%$ of variance, whereas 1 -factor solution explained $42 \%$. Reliability for the 1 -factor solution was assessed with the rating of the 4 negatively worded items (no. 5 to 8 ) reversed (e.g., from 0 to 6 , and from 6 to 0 ). Chronbach's $\alpha$ of the total 10 -item scale was 0.862 .

Table 3. Comparison of Item Scores between the Current Daily-only Treatment and an Imaginary Daily-and-Weekly Treatment.

\begin{tabular}{|c|c|c|c|c|}
\hline & \multicolumn{2}{|l|}{ Score of agreement } & \multicolumn{2}{|c|}{ Difference between two treatments } \\
\hline & $\begin{array}{l}\text { Current daily-only } \\
\text { treatment }\end{array}$ & $\begin{array}{l}\text { Imaginary } \\
\text { daily-and-weekly } \\
\text { treatment }\end{array}$ & $\mathrm{p}$ value & Effect size $d$ \\
\hline 1. Convenience & $4.5 \pm 1.5$ & $4.0 \pm 2.1$ & $\mathrm{p}<0.001$ & 0.20 \\
\hline 2. Encouragement of adherence & $4.7 \pm 1.4$ & $4.3 \pm 1.8$ & $\mathrm{p}<0.001$ & 0.21 \\
\hline 3. Control over diseases & $4.7 \pm 1.3$ & $3.9 \pm 1.7$ & $\mathrm{p}<0.001$ & 0.44 \\
\hline 4. Feeling of healthiness & $4.2 \pm 1.6$ & $3.9 \pm 1.7$ & $\mathrm{p}<0.001$ & 0.20 \\
\hline 5. Troublesomeness & $1.9 \pm 1.9$ & $2.2 \pm 1.9$ & $\mathrm{p}=0.003$ & 0.10 \\
\hline 6. Fear of forgetting to take medicines & $2.0 \pm 2.0$ & $3.1 \pm 2.2$ & $\mathrm{p}<0.001$ & 0.41 \\
\hline 7. Suspicion about efficacy & $2.1 \pm 1.9$ & $2.9 \pm 1.9$ & $\mathrm{p}<0.001$ & 0.38 \\
\hline 8. Undesirably weakened disease awareness & $1.8 \pm 1.7$ & $2.6 \pm 1.9$ & $\mathrm{p}<0.001$ & 0.41 \\
\hline 9. Satisfaction & $4.4 \pm 1.4$ & $3.4 \pm 2.0$ & $\mathrm{p}<0.001$ & 0.43 \\
\hline 10. Hope for receiving the treatment & $4.3 \pm 1.6$ & $3.2 \pm 2.2$ & $\mathrm{p}<0.001$ & 0.40 \\
\hline Total satisfaction score & $43 \pm 11$ & $36 \pm 13$ & $\mathrm{p}<0.001$ & 0.49 \\
\hline
\end{tabular}

Table 4. Expected Satisfaction with Imaginary Daily-and-Weekly Treatment.

\begin{tabular}{|c|c|c|c|c|}
\hline $\begin{array}{l}\text { Quintiles of } \\
\text { satisfaction score } \\
\text { for the current } \\
\text { daily-only } \\
\text { treatment }\end{array}$ & $\begin{array}{l}\text { Total satisfaction score } \\
\text { for the current } \\
\text { daily-only treatment } \\
\text { (current satisfaction) } \\
S_{\mathrm{c}}\end{array}$ & $\begin{array}{l}\text { Total satisfaction score for } \\
\text { an imaginary } \\
\text { daily-and-weekly } \\
\text { treatment } \\
\text { (expected satisfaction) } \\
S_{\mathrm{w}}\end{array}$ & $\begin{array}{l}\text { Difference of total satisfaction } \\
\text { score (imaginary } \\
\text { daily-and-weekly treatment vs. } \\
\text { current daily-only treatment) } \\
S_{\mathrm{w}}-S_{\mathrm{c}}\end{array}$ & $\begin{array}{l}\text { Cases giving a higher } \\
\text { total score for an } \\
\text { imaginary } \\
\text { daily-and-weekly } \\
\text { treatment } \\
\left(S_{\mathrm{w}}>S_{\mathrm{c}}\right) \\
\end{array}$ \\
\hline Q1 $(n=195)$ & $28.3 \pm 3.9$ & $31.7 \pm 9.9$ & 3.4 (95\% CI: 1.9 to 4.9$)$ & $115(59 \%)$ \\
\hline Q2 $(n=178)$ & $35.3 \pm 1.7$ & $33.4 \pm 9.8$ & $-1.9(95 \%$ CI: -3.4 to -0.5$)$ & $72(40 \%)$ \\
\hline Q3 $(n=209)$ & $42.0 \pm 2.3$ & $35.4 \pm 11.8$ & $-6.7(95 \%$ CI: -8.3 to -5.1$)$ & $60(29 \%)$ \\
\hline $\mathrm{Q} 4(\mathrm{n}=195)$ & $50.4 \pm 2.3$ & $38.9 \pm 12.0$ & $-11.5(95 \%$ CI: -13.2 to -9.8$)$ & $28(14 \%)$ \\
\hline Q5 $(n=196)$ & $58.3 \pm 1.8$ & $40.7 \pm 16.0$ & $-17.6(95 \%$ CI: -19.8 to -15.4$)$ & $16(8 \%)$ \\
\hline $\mathrm{p}$ for trend & $\mathrm{p}<0.001$ & $\mathrm{p}<0.001$ & $\mathrm{p}<0.001$ & $\mathrm{p}<0.001$ \\
\hline
\end{tabular}

The possible range of the satisfaction score was from 0 to 60 .

treatment; the standardized partial regression coefficient $\beta$ was -0.012 for the number (i.e. the kind) of medications $(\mathrm{p}=$ $0.809),-0.036$ for the frequency of dosing $(\mathrm{p}=0.381)$, and the combined use of self-injections $(\beta=0.007, p=0.837)$, respectively. We also confirmed that the total satisfaction score for the imaginary daily-and-weekly treatment was not significantly correlated with age $(\mathrm{r}=0.051, \mathrm{p}=0.115)$. Neither was it significantly associated with diabetes mellitus ( $\mathrm{p}=$ $0.438)$, dyslipidemia $(\mathrm{p}=0.948)$, circulatory diseases $(\mathrm{p}=$ 0.511 ), or hyperuricemia $(\mathrm{p}=0.601)$. In addition, it was not significantly correlated with the control levels; the correlation coefficient $r$ was $0.020(p=0.588)$ for hemoglobin A1c levels and $0.053(\mathrm{p}=0.312)$ for fasting plasma glucose levels in patients with diabetes mellitus, $-0.014(\mathrm{p}=0.751)$ for low- 
density lipoprotein cholesterol levels and -0.009 ( $\mathrm{p}=0.886)$ for triglycerides levels in patients with dyslipidemia, -0.040 $(\mathrm{p}=0.371)$ for systolic blood pressure and $-0.034(\mathrm{p}=0.444)$ for diastolic blood pressure in patients with hypertension, and $-0.155(\mathrm{p}=0.079)$ for uric acid levels in patients with hyperuricemia.

\section{Discussion}

The current study indicated that treatment satisfaction would be, on average, attenuated in patients treated with multiple daily oral medications for chronic diseases if one of the oral medications was changed to a once-weekly one. Patients giving a higher score for the imaginary daily-andweekly treatment than for the current daily-only treatment were more prevalent in the subgroup with a lower score for the current daily-only treatment.

The risk analysis for the current treatment satisfaction revealed that a higher daily frequency of dosing and combined use of self-injections were negatively associated with the treatment satisfaction, indicating that the complexity of the dosing regimens would be less satisfying. Indeed, these complicated regimens are reported to be linked with nonadherence (4).

Drug regimens with reduced dosing frequencies are believed to be preferred by patients, which might result in improved treatment satisfaction. Based on this belief, onceweekly oral medications have been developed (5), and onceweekly bisphosphonates are one of the successful precedents $(8,9)$. However, the current findings showed that the majority of the patients treated with polypharmacy for lifestyle-related chronic diseases gave a lower satisfaction score to the imaginary daily-and-weekly treatment than the current daily-only treatment, indicating that they did not favor a regimen using a once-weekly medication together.

Although the true underlying reason for this unexpected finding is unknown, one possible explanation is that the medications for lifestyle-related chronic diseases currently available are not as troublesome to consume as bisphosphonates. Bisphosphonates are not easy to take; patients must take a bisphosphonate after waking up in the morning, with a full glass (i.e. around $180 \mathrm{~mL}$, and not less) of water, and no other drink (including water rich in minerals), at least 30 minutes before eating a meal, drinking a beverage, or taking any other medicine. They must also sit or stand (not lie down) for at least 30 minutes after taking it. The troublesomeness of taking a bisphosphonate may encourage the preference for once-weekly dosing. By contrast, the currently available daily medications for lifestyle-related chronic diseases are not as troublesome to take. Relatively, the troublesomeness of combined use of a once-weekly oral medication plus daily ones would stand out.

A previous questionnaire-based study reported that patients were generally positive about once-weekly medications (10). However, the researchers drew the patients' responses by presenting the hypothesis that patients could change all oral medications for a once weekly medication if they wished. This situation is in contrast to the current clinical setting, in which only a few kinds of once-weekly medications are available at present and one once-weekly medication cannot be a substitute for polypharmacy. As such, once-weekly medications must be combined with daily medications for the time being. Our study demonstrated that, in such a real-world situation, only a limited population would draw an expected advantage, i.e. improved satisfaction, from a daily-and-weekly combination treatment.

The per-item analysis (Table 3 ) showed that inferiority of the imaginary daily-and-weekly treatment to the current daily-only treatment was observed in various aspects. Patients did not regard the treatment as all that simple, and were suspicious about the drug efficacy. An unfavorable impression of a newly started medication may lead to reluctance to take the medication (11-13). When medical staff make a well-meant attempt to switch a daily medication to a weekly one in non-adherent patients with polypharmacy, they should be aware of this potential psychological barrier to the daily-and-weekly treatment. The patients' preformed impression of the daily-and-weekly treatment is often poor, and an attempt to alter the regimen might further deteriorate the medication adherence, contrary to expectations.

However, we must not overlook the fact that a third of the population gave a higher score for the imaginary daily-andweekly treatment than the current daily-only treatment, suggesting that a proposal to switch one daily medication to a weekly one would draw favorable responses from these patients. Unfortunately, the current study could not detect any significant clinical predictor for the total satisfaction score for the imaginary daily-and-weekly treatment, meaning that it would be difficult to predict the expected satisfaction based on the patients' clinical backgrounds.

Given that the lowest quintile of the current treatment satisfaction was more likely to give a relatively high satisfaction score for the imaginary daily-and-weekly treatment, the risk factors for dissatisfaction with the current daily-only treatment (i.e. the frequency of dosing and the combined use of self-injections) would be clinically useful as passive markers for relative satisfaction with the daily-and-weekly treatment. Indeed, when we performed a multivariate logistic regression analysis, the frequency of dosing and the combined use of self-injections were significantly associated with a higher satisfaction score for the imaginary daily-andweekly treatment than the current daily-only treatment (both $\mathrm{p}<0.05$ ) (data not shown). However, it is of note that these variables were only risk factors for dissatisfaction with the current daily-only treatment and for a relatively high satisfaction score for the imaginary daily-and-weekly treatment; they were not direct predictors for the absolute satisfaction with the imaginary daily-and-weekly treatment. The psychological factors involved in the expected satisfaction with the daily-and-weekly treatment must be thoroughly investigated. A deeper understanding of the patient perspective on the combination therapy of once-weekly oral medication plus 
daily ones would promote the improvement of treatment satisfaction and medication adherence. A more detailed survey regarding the patient perspective on combination therapy of once-weekly medications will be needed.

Several limitations associated with the present study warrant mention. First, we only surveyed the patients' expected satisfaction with an imaginary treatment using a onceweekly medication and did not actually administer the treatment to the patients. The real satisfaction when they actually receive the treatment remains unknown. However, given that patients' perspectives could substantially influence medication adherence, the current findings may still be of use in initiating a once-weekly medication. Second, the current questionnaire did not survey the patient perspective on the imaginary therapy with all their medications changed to once-weekly ones. We were therefore unable to compare that perspective with the current satisfaction or the dailyand-weekly treatment. Third, the current study did not include patients who already took once-weekly medications. Fourth, the current study did not prospectively survey the patients' medication adherence. Retrospectively, the data on the pill count-based medication adherence were available from medical records in 363 diabetic patients (36\%). In this limited population, medication adherence was distributed as $94 \% \pm 10 \% ; 165$ patients $(46 \%)$ had $100 \%$ adherence, 132 (36\%) had 90-99\%, 43 (12\%) had 80-89\%, and $23(6 \%)$ had $\leq 79 \%$ adherence. The medication adherence was significantly associated with a total satisfaction score for the current daily-only treatment $(\mathrm{r}=0.120, \mathrm{p}=0.022)$ but was not correlated with that for the imaginary daily-and-weekly treatment $(\mathrm{r}=0.003, \mathrm{p}=0.960)$. However, this finding was from a limited population, and whether or not this finding is representative of a trend in the whole population remains unknown. Future studies prospectively and thoroughly investigating medication adherence in the whole study population will be needed to confirm the association with the patient perspective and the medication adherence. Fifth, only Japanese patients were included in the current study. Medication insurance systems, cultures, relative socioeconomic status, and other confounding factors might influence the attitude toward treatments. Future studies with populations of other ethnicities will be needed to validate the current findings.

In conclusion, the current study indicated that treatment satisfaction would be on average attenuated in patients treated with more than one daily oral medication for chronic diseases if one of the oral medications was changed to a once-weekly one. Improvements in the satisfaction were less expected in the subgroup more satisfied with the current daily-only treatment.

Author's disclosure of potential Conflicts of Interest (COI). Mitsuyoshi Takahara: Research funding, Takeda Pharmaceutical and MSD. Toshihiko Shiraiwa: Honoraria, Takeda Pharmaceutical. Naoto Katakami: Research funding, Takeda Pharmaceutical and MSD. Taka-aki Matsuoka: Research funding, Takeda Pharmaceutical and MSD. Iichiro Shimomura: Honoraria, Takeda Pharmaceutical and MSD; Research funding, Takeda Pharmaceutical and MSD.

\section{Acknowledgement}

The current study was self-funded. The authors thank the following medical staff who contributed to collecting questionnaires: Ayaka Nakaoka, Shiori Ozaki, Yuki Katada, Mami Fujimoto, and Yukari Morioka in Shiraiwa Medical Clinic.

\section{References}

1. Ho PM, Rumsfeld JS, Masoudi FA, et al. Effect of medication nonadherence on hospitalization and mortality among patients with diabetes mellitus. Arch Intern Med 166: 1836-1841, 2006.

2. Sokol MC, McGuigan KA, Verbrugge RR, Epstein RS. Impact of medication adherence on hospitalization risk and healthcare cost. Med Care 43: 521-530, 2005.

3. Barbosa CD, Balp MM, Kulich K, Germain N, Rofail D. A literature review to explore the link between treatment satisfaction and adherence, compliance, and persistence. Patient Prefer Adherence 6: 39-48, 2012.

4. Coleman CI, Limone B, Sobieraj DM, et al. Dosing frequency and medication adherence in chronic disease. J Manag Care Pharm 18: 527-539, 2012.

5. Garber AJ. The importance of incretin therapies for managing type 2 diabetes. Lancet Diabetes Endocrinol 2: 95-97, 2014.

6. Inagaki N, Onouchi H, Maezawa H, Kuroda S, Kaku K. Onceweekly trelagliptin versus daily alogliptin in Japanese patients with type 2 diabetes: a randomised, double-blind, phase 3, noninferiority study. Lancet Diabetes Endocrinol 3: 191-197, 2015.

7. Sheu WH, Gantz I, Chen M, et al. Safety and efficacy of omarigliptin (MK-3102), a novel once-weekly DPP-4 inhibitor for the treatment of patients with type 2 diabetes. Diabetes Care 38: 2106-2114, 2015.

8. Simon JA, Lewiecki EM, Smith ME, Petruschke RA, Wang L, Palmisano JJ. Patient preference for once-weekly alendronate 70 $\mathrm{mg}$ versus once-daily alendronate $10 \mathrm{mg}$ : a multicenter, randomized, open-label, crossover study. Clin Ther 24: 1871-1886, 2002.

9. Kendler D, Kung AW, Fuleihan Gel H, et al. Patients with osteoporosis prefer once weekly to once daily dosing with alendronate. Maturitas 48: 243-251, 2004.

10. Polonsky WH, Fisher L, Hessler D, Bruhn D, Best JH. Patient perspectives on once-weekly medications for diabetes. Diabetes Obes Metab 13: 144-149, 2011.

11. World Health Organization. Adherence to long-term therapies: evidence for action. WHO, Geneva, 2003.

12. Casula M, Tragni E, Catapano AL. Adherence to lipid-lowering treatment: the patient perspective. Patient Prefer Adherence 6: 805814, 2012.

13. Brown MT, Bussell JK. Medication adherence: WHO cares? Mayo Clin Proc 86: 304-314, 2011.

The Internal Medicine is an Open Access article distributed under the Creative Commons Attribution-NonCommercial-NoDerivatives 4.0 International License. To view the details of this license, please visit (https://creativecommons.org/licenses/ by-nc-nd/4.0/).

(C) 2017 The Japanese Society of Internal Medicine

http://www.naika.or.jp/imonline/index.html 